\title{
A converse to global exponential stability of a class of difference equations
}

\author{
Yinghua Fu ${ }^{1,2 *}$, Qunfei Zhao ${ }^{2}$ and Lisheng Wang ${ }^{2}$
}

"Correspondence: fuyh@usst.edu.cn ${ }^{1}$ School of Optical-Electrical and Computer Engineering, University of Shanghai for Science and Technology, Shanghai, 200093, P.R. China

${ }^{2}$ Department of Automation, Shanghai Jiao Tong University, Shanghai, 200240, P.R. China

\begin{abstract}
This paper discusses the global exponential stability of a class of difference equations. Sufficient and necessary conditions for the global exponential stability are derived. Particularly, the equivalent relationship between the global exponential stability of difference equations and the contractive property of the nonlinear operator of the systems is shown.
\end{abstract}

Keywords: global exponential stability; exponential bound; difference equations; strongly equivalent metric

\section{Introduction}

In mathematics and automatic control, an equation system is discussed as discrete or continuous one with the type of input variables and linear or nonlinear one according to the relationship between the input and output. The stability of the equation systems always catches researchers' eyes. The Lyapunov function is important for stability theory and control theory, and its existence is a necessary and sufficient condition of stability for many differential equation systems. Linear systems received more attention than nonlinear systems because of simplicity and controllability, and many good results are shown in the textbooks and literature. Bastinec [1] gives the sufficient condition for exponential stability and estimation of solutions of linear differential systems with delay-dependence. Nonlinear systems are of interest to researchers and engineers because most physical systems are inherently nonlinear in nature and difficult to solve. The converse Lyapunov problem on the stability of nonlinear discrete dynamical systems is developed and studied by many researchers (Gordon [2]; Ortega [3]; Mousa et al. [4]; Jiang and Wang [5]; Wang et al. [6]). Diblik [7] considers a particular critical case and gives conditions for the stability of a zero solution of difference systems with quadratic nonlinearities using the method of Lyapunov functions and derives classes of stable systems. Furthermore, the author estimates the stability domains as well. Berezansky [8] considers the asymptotic convergence of solutions in discrete and difference equations with delay and gives strong sufficient conditions of the asymptotic convergence of all solutions without computing the limits of the solutions as $n \rightarrow \infty$. It demonstrates that a difference equation is of exponential stability in $R^{n}$ if and only if there is a Lyapunov function which is an abstract presentation of system energy (Mousa et al. [4]; Jiang and Wang [5]; Wang et al. [9]; Wang et al. [10]). The inverse problem of exponential stability instead of the inverse Lyapunov function has been considered from a different view in the recent literature (Wang et al. [9]; Wang and Xu [11]).

C 2013 Fu et al: licensee Springer. This is an Open Access article distributed under the terms of the Creative Commons Attribution License (http://creativecommons.org/licenses/by/2.0), which permits unrestricted use, distribution, and reproduction in any medium, provided the original work is properly cited. 
Consider the following difference equation (Wang et al. [9]; Wang and Xu [11]):

$$
x_{k+1}=T x_{k}, \quad x_{0} \in D, k=0,1,2, \ldots
$$

where $D \subset R^{n}$ is a bounded closed set, $T: D \rightarrow D$ is nonlinear and continuously differentiable in $D$. Then $T$ is a Lipschitz continuous operator. $x^{*} \in D$ is the unique equilibrium point (fixed point). Wang et al. [9], Wang and Xu [11] show that system (1) is of exponential stability in $D$ if and only if $T$ is contractive in $D$ with a certain strongly equivalent metric and also present a converse not only to the exponential stability of system (1) different from the Lyapunov function, but also to the Banach contraction theorem different from the ones in Meyers [12], Leader [13], Opoitsev [14]. In other words, the two works show that $T$ is contractive in $D$ with a certain strongly equivalent metric if its iterative sequence $\left\{x_{k}\right\}$ exponentially converges to $x^{*}$ for any initial point $x_{0} \in D$. The result is helpful to understand the exponential stability of $x^{*}$ from a different view. However, Wang et al. [9], Wang and $\mathrm{Xu}$ [11] only consider nonlinear discrete dynamical systems in the bounded set $D$. The problem whether the result can be extended to difference equations in an unbounded set still remains open. It is hard to answer for general difference equations.

This paper focuses on a class of important difference equations in $R^{n}$ shown as follows:

$$
x_{k+1}=T x_{k}, \quad x_{0} \in R^{n}, k=0,1,2, \ldots,
$$

where $x^{*} \in R^{n}$ is the unique equilibrium point and $T: R^{n} \rightarrow R^{n}$ is a continuous differentiable nonlinear operator in $R^{n}$, and $\lim _{\|x\| \rightarrow \infty} \sup \left\|T^{\prime}(x)\right\|=\lambda<1$ holds for a positive constant $\lambda$ (e.g., $0<\lambda<1)$. System (2) includes the discrete-time neural network model in Wang and $\mathrm{Xu}$ [10] and difference equations in Wang et al. [9], Wang and $\mathrm{Xu}$ [11] as the special cases. The exponential stability of system (2) is developed and necessary and sufficient conditions to be exponentially stable in $R^{n}$ (namely, globally exponentially stable) are derived. Particularly, we give the result that system (2) is of global exponential stability if and only if $T$ is contractive in $R^{n}$ with a certain strongly equivalent metric. A simple method to distinguish global exponential stability from global asymptotic stability is proposed. The results in this paper are helpful to discover some properties of the global exponential stability of system (2).

\section{Definition and main results}

A nonlinear operator $T$ is Lipschitz continuous if $\|T x-T y\| \leq M \cdot\|x-y\|$ is satisfied for any $x, y \in D$ and the given constant $M(M>0)$, and contractive in $E \subset R^{n}$ with a strongly equivalent metric $d(\cdot, \cdot)$ if there is a constant $0<\beta<1$ such that $d(T x, T y) \leq \beta \cdot d(x, y)$ holds for any $x, y \in E$. A metric function $d(\cdot, \cdot): E \times E \rightarrow R^{+}$is a strongly equivalent metric with the vector norm $\|\cdot\|$ (or strongly equivalent with $\|\cdot\|$ ) in the set $E \subset R^{n}$ if there are two constants $C_{2} \geq C_{1}>0$ such that for any $x, y \in E$, the following inequalities hold (Wang and $\mathrm{Xu}[11])$ :

$$
C_{1} \cdot d(x, y) \leq\|x-y\| \leq C_{2} \cdot d(x, y)
$$

The equilibrium point of some operator (for example, (1) or (2)) is globally asymptotically stable in $R^{n}$ if it is stable in the sense of Lyapunov and $\lim _{k \rightarrow \infty} x_{k}=x^{*}$ holds for any $x_{0} \in R^{n}$. 
And it is exponentially stable in the set $E \subset R^{n}$ if there are constants $M>0$ and $0<\alpha<1$ such that for any initial point $x_{0}$ in $E$ and the positive integer $k$,

$$
\left\|x_{k}-x^{*}\right\| \leq M \cdot \alpha^{k} \cdot\left\|x_{0}-x^{*}\right\|
$$

Let $\Phi$ be the set of all equivalent norms of $\|\cdot\|, \Omega$ be the set of all strongly equivalent metrics of $\|\cdot\|$ in $E \subset R^{n}$ and $\Theta$ be the set of all topologically equivalent metrics of $\|\cdot\|$ in $E$. Then the inclusion relationship $\Phi \subset \Omega \subset \Theta$ is satisfied. In other words, each equivalent norm of $\|\cdot\|$ is also a strongly equivalent metric, and each strongly equivalent metric is a topologically equivalent metric. Generally, $T$ maybe is not contractive in $R^{n}$ with any equivalent norm even if system (2) is of exponential stability. Besides, system (2) is possibly not of exponential stability in $R^{n}$ even if $T$ is contractive with certain topologically equivalent metric (Wang and $\mathrm{Xu}$ [11]). In this paper, we show that strongly equivalent metrics are a class of appropriate equivalent metric functions describing the exponential stability of system (2).

Suppose $F: E \rightarrow E$ is a Lipschitz continuous nonlinear operator in the arbitrary subset $E \subset R^{n}$. Then the lub Lipschitz constants of $F$ with $\|\cdot\| \in \Phi$ and $d(\cdot, \cdot) \in \Omega$ are defined as follows, respectively (Wang and $\mathrm{Xu}$ [11]; Soderlind [15]):

$$
L_{\|\|}(F, E)=\sup _{x, y \in E, x \neq y} \frac{\|F x-F y\|}{\|x-y\|}, \quad L_{d}(F, E)=\sup _{x, y \in E, x \neq y} \frac{d(F x, F y)}{d(x, y)}
$$

where $L_{\|\|}(F, E)$ and $L_{d}(F, E)$ are two functions determined by $F, E$ and $\|\cdot\|$ or $d(\cdot, \cdot)$. Hence, for any $x, y \in E$, one obtains

$$
\|F x-F y\| \leq L_{\|}(F, E) \cdot\|x-y\|, \quad d(F x, F y) \leq L_{d}(F, E) \cdot d(x, y) .
$$

Wang and Xu [11] give the result that $\lim _{k \rightarrow \infty} L_{\|\|}\left(F^{k}, E\right)^{1 / k}$ and $\lim _{k \rightarrow \infty} L_{d}\left(F^{k}, E\right)^{1 / k}$ exist and the following formulas hold:

$$
\lim _{k \rightarrow \infty} L_{d}\left(F^{k}, E\right)^{1 / k}=\lim _{k \rightarrow \infty} L_{\|\|}\left(F^{k}, E\right)^{1 / k}=\inf _{k} L_{\|\|}\left(F^{k}, E\right)^{1 / k} .
$$

Denote $\operatorname{Lip}(F, E)=\lim _{k \rightarrow \infty} L_{\|} \|\left(F^{k}, E\right)^{1 / k}$. Then $\operatorname{Lip}(T, E)$ is a constant independent of different equivalent norms or strongly equivalent metrics. $L_{\|\|}(F, E)$ and $\operatorname{Lip}(T, E)$ can be regarded as nonlinear generalizations of the matrix norm and matrix spectral radius. By Wang and $\mathrm{Xu}[11]$, one can have

$$
\operatorname{Lip}(F, E)=\inf _{d(\cdot, \cdot) \in \Omega} L_{d}(F, E)
$$

Namely, $\operatorname{Lip}(T, E)$ is a minimum Lipschitz constant. Furthermore, $F$ is contractive in $E$ with respect to a certain strongly equivalent metric in $\Omega$ if and only if $\operatorname{Lip}(F, E)<1$ or $L_{\|\|}\left(T^{m}, E\right)<1$ holds for a positive integer $m$.

Lemma 1 In system (2), $L_{\|\|}\left(T, R^{n}\right)<\infty$ holds. And for any $x \in R^{n}$ and a positive integer $j$, one obtains $\left\|\left(T^{j}\right)^{\prime}(x)\right\| \leq L_{\|\|}\left(T^{j}, R^{n}\right) \leq L_{\|\|}\left(T, R^{n}\right)^{j}$. 
Hence, the nonlinear operator $T$ in system (2) is Lipschitz continuous in $R^{n}$. The main results of this paper are presented below.

Theorem 1 In system (2), the following five conditions are equivalent:

(A) $x^{n}$ is globally exponentially stable in $R^{n}$.

(B) $x^{*}$ is globally asymptotically stable in $R^{n}$ and $\rho\left(T^{\prime}\left(x^{*}\right)\right)<1$.

(C) $\operatorname{Lip}\left(T, R^{n}\right)<1$.

(D) $T$ is contractive in $R^{n}$ with certain strongly equivalent metrics of \|\| .

(E) The power of $T$ is contractive in $R^{n}$ with the norm \|\| , i.e., there exist a positive integer $m$ and a constant $0<\beta<1$ such that $\left\|T^{m} x-T^{m} y\right\| \leq \beta \cdot\|x-y\|$ holds for any $x, y \in R^{n}$.

As long as system (2) is of global exponential stability, we have $\operatorname{Lip}\left(T, R^{n}\right)<\alpha$, and for any sufficiently small $\varepsilon \in\left(0,1-\operatorname{Lip}\left(T, R^{n}\right)\right), \operatorname{Lip}\left(T, R^{n}\right)+\varepsilon$ is an exponential bound. Here, $\alpha=\max \left\{\lambda, \rho\left(T^{\prime}\left(x^{*}\right)\right)\right\}$ and $\rho\left(T^{\prime}\left(x^{*}\right)\right)$ is the spectral radius of the Jacobian matrix $T^{\prime}\left(x^{*}\right)$.

Theorem 1 shows that we can distinguish global exponential stability from global asymptotic one for system (2) by calculating the spectral radius of the Jacobian matrix at the equilibrium point $x^{*}$, namely, $\rho\left(T^{\prime}\left(x^{*}\right)\right)$. It tells us that the global exponential stability of system (2) is equivalent to the contractive property of the nonlinear operator $T$ with certain strongly equivalent metrics of the norm $\|\cdot\|$. It actually describes a new converse to the Banach contraction theorem differing from the ones in Meyers [12], Leader [13], Opoitsev [14].

Theorem 2 In system (2), assume $\lim _{\|x\| \rightarrow \infty} \sup \left\|T^{\prime}(x)\right\| \leq \rho\left(T^{\prime}\left(x^{*}\right)\right)$. If $x^{*}$ is globally exponentially stable in $R^{n}$, then $\operatorname{Lip}\left(T, R^{n}\right)=\rho\left(T^{\prime}\left(x^{*}\right)\right)$ and $\rho\left(T^{\prime}\left(x^{*}\right)\right)$ is the infimum of all exponential bounds of convergent trajectories. Particularly,

$$
\left\|x_{k}-x^{*}\right\| \leq \frac{C(\varepsilon)_{2}}{C(\varepsilon)_{1}} \cdot\left(\rho\left(T^{\prime}\left(x^{*}\right)\right)+\varepsilon\right)^{k} \cdot\left\|x_{0}-x^{*}\right\|
$$

holds for any $\varepsilon>0$ and $x_{0} \in R^{n}$, where $C(\varepsilon)_{2}>C(\varepsilon)_{1}>0$ are the same constants as in Theorem 1 .

Theorem 2 shows that exponential bounds of convergent trajectories describing the global property of trajectory motion in the whole space actually can be determined by the local information at the equilibrium point, namely $\rho\left(T^{\prime}\left(x^{*}\right)\right)$.

\section{Proofs}

In this section, the proofs of results and theorems will be given.

Proof of Lemma 1 Since $\lim _{\|x\| \rightarrow \infty} \sup \left\|T^{\prime}(x)\right\|=\lambda$, for any $\varepsilon>0$, there is $b>0$ such that $\left\|T^{\prime}(x)\right\| \leq \lambda+\varepsilon$ as $\left\|x-x^{*}\right\| \geq b$. Let $F(b)=\left\{x \in R^{n}:\left\|x-x^{*}\right\| \leq b\right\}$. Since $T$ is continuously differentiable in $R^{n},\left\|T^{\prime}(x)\right\|$ is a continuous function in the compact set $F(b)$. Thus, we have $\sup _{z \in F(b)}\left\|T^{\prime}(z)\right\|<\infty$. This implies that $M=\sup _{z \in R^{n}}\left\|T^{\prime}(z)\right\|<\infty$ holds. From the mid-value theorem, for any $x, y \in R^{n}$, one obtains

$$
\|T x-T y\| \leq \sup _{z \in R^{n}}\left\|T^{\prime}(z)\right\| \cdot\|x-y\| \leq M \cdot\|x-y\| .
$$


This implies that $L_{\|\|}\left(T, R^{n}\right) \leq M<\infty$ holds. Since $T^{j}$ is continuously differentiable, with the definition of the Gateaux differential, for any $x \in R^{n}$ and any $h \in R^{n}$ with $\|h\|=1$,

$$
\left\|\left(T^{j}\right)^{\prime}(x) \cdot h\right\|=\lim _{t \rightarrow 0^{+}} \frac{\left\|T^{j}(x+t \cdot h)-T^{j} x\right\|}{t}=\lim _{t \rightarrow 0^{+}} \frac{\left\|T^{j}(x+t \cdot h)-T^{j} x\right\|}{\|t \cdot h\|} \leq L_{\|\|}\left(T^{j}, R^{n}\right) .
$$

This implies that

$$
\left\|\left(T^{j}\right)^{\prime}(x)\right\|=\sup _{\|h\|=1}\left\|\left(T^{j}\right)^{\prime}(x) \cdot h\right\| \leq L_{\|\|}\left(T^{j}, R^{n}\right) \leq L_{\|\|}\left(T, R^{n}\right)^{j} .
$$

The proof is done.

Proof of Theorem 1 The equivalent relationships between (C), (D) and (E) can be easily gotten from Wang and Xu [11]. And it is also easy to deduce (B) from (A). Next, we mainly prove $(\mathrm{C}) \Rightarrow(\mathrm{A})$ and $(\mathrm{B}) \Rightarrow(\mathrm{C})$.

Firstly, prove $(\mathrm{C}) \Rightarrow(\mathrm{A})$.

If $\operatorname{Lip}\left(T, R^{n}\right)<1$, then system (2) is of global exponential stability in $R^{n}$. With equation (6), for any sufficiently small $\varepsilon \in\left(0,1-\operatorname{Lip}\left(T, R^{n}\right)\right)$, there is a strongly equivalent metric $d_{\varepsilon}(\cdot, \cdot) \in \Omega$ such that $L_{d_{\varepsilon}}\left(T, R^{n}\right) \leq \operatorname{Lip}\left(T, R^{n}\right)+\varepsilon<1$. Assume that $d_{\varepsilon}(\cdot, \cdot)$ and $\|\cdot\|$ have the following strongly equivalent relationships:

$$
C(\varepsilon)_{1} \cdot d_{\varepsilon}(x, y) \leq\|x-y\| \leq C(\varepsilon)_{2} \cdot d_{\varepsilon}(x, y) \quad \forall x, y \in R^{n},
$$

where $C(\varepsilon)_{2}>C(\varepsilon)_{1}>0$ are two constants. For any positive integer $k$ and any $x \in R^{n}$, we have

$$
\begin{aligned}
\left\|T^{k} x-x^{*}\right\| & =\left\|T^{k} x-T^{k} x^{*}\right\| \leq C(\varepsilon)_{2} \cdot d_{\varepsilon}\left(T^{k} x, T^{k} x^{*}\right) \\
& \leq C(\varepsilon)_{2} \cdot L_{d_{\varepsilon}}\left(T, R^{n}\right)^{k} \cdot d_{\varepsilon}\left(x, x^{*}\right) \\
& \leq C(\varepsilon)_{2} \cdot\left(\operatorname{Lip}\left(T, R^{n}\right)+\varepsilon\right)^{k} \cdot d_{\varepsilon}\left(x, x^{*}\right) \\
& \leq \frac{C(\varepsilon)_{2}}{C(\varepsilon)_{1}} \cdot\left(\operatorname{Lip}\left(T, R^{n}\right)+\varepsilon\right)^{k} \cdot\left\|x-x^{*}\right\| .
\end{aligned}
$$

Thus, system (2) is of global exponential stability in $R^{n}$ with the exponential bound $\operatorname{Lip}\left(T, R^{n}\right)+\varepsilon$.

Secondly, prove (B) $\Rightarrow$ (C); namely, if system (2) is of global exponential stability in $R^{n}$ and $\rho\left(T^{\prime}\left(x^{*}\right)\right)<1$, then $\operatorname{Lip}\left(T, R^{n}\right)<1$.

Since $\lim _{\|x\| \rightarrow \infty} \sup \left\|T^{\prime}(x)\right\|=\lambda \leq \alpha<1$, for any $\varepsilon \in(0,1-\alpha)$, there is a constant $b>0$ such that $\left\|T^{\prime}(x)\right\| \leq \alpha+\varepsilon<1$ as $\left\|x-x^{*}\right\| \geq b$. Let $E(b)=\left\{x \in R^{n}:\left\|x-x^{*}\right\|>b\right\}, F(b)=\{x \in$ $\left.R^{n}:\left\|x-x^{*}\right\| \leq b\right\}, B(b)=\left\{x \in R^{n}:\left\|x-x^{*}\right\| \leq 2 b\right\}$. For any $z \in E(b)$ and any given positive integer $m$, if $T^{i} z \in E(b)$ holds for any positive integer $i=0,1, \ldots, m-1$, then by the chainrule of derivative, one gets

$$
\left\|\left(T^{m}\right)^{\prime}(z)\right\|=\left\|\prod_{i=1}^{m} T^{\prime}\left(T^{m-i} z\right)\right\| \leq \prod_{i=1}^{m}\left\|T^{\prime}\left(T^{m-i} z\right)\right\| \leq(\alpha+\varepsilon)^{m} .
$$

For the fixed $\varepsilon \in(0,1-\alpha)$, there exists an equivalent norm $\|\cdot\|_{\varepsilon}$ such that the subordinated matrix norm $\left\|T^{\prime}\left(x^{*}\right)\right\|_{\varepsilon} \leq \rho\left(T^{\prime}\left(x^{*}\right)\right)+\varepsilon / 2$. Since $T$ is continuously differentiable, $\left\|T^{\prime}(x)\right\|_{\varepsilon}$ 
is a continuous function of $x \in R^{n}$. It implies that there exists a spherical neighborhood $U(r)=\left\{x \in R^{n}:\left\|x-x^{*}\right\|_{\varepsilon} \leq r\right\}$ such that for any $x \in U(r)$,

$$
\left\|T^{\prime}(x)\right\|_{\varepsilon} \leq\left\|T^{\prime}\left(x^{*}\right)\right\|_{\varepsilon}+\varepsilon / 2 \leq \rho\left(T^{\prime}\left(x^{*}\right)\right)+\varepsilon .
$$

By the mid-value theorem, for any $x, y \in U(r)$, we have

$$
\|T x-T y\|_{\varepsilon} \leq \sup _{z \in U(r)}\left\|T^{\prime}(z)\right\|_{\varepsilon} \cdot\|x-y\|_{\varepsilon} \leq\left(\rho\left(T^{\prime}\left(x^{*}\right)\right)+\varepsilon\right) \cdot\|x-y\|_{\varepsilon}
$$

If system (2) is of global exponential stability in $R^{n}$, it is globally uniformly asymptotically stable in $R^{n}$ as well (Elaydi [16]). Therefore, there exists a positive integer $N$ such that for any positive integer $k \geq N$ and any $x \in B(b), T^{k} x \in U(r)$ holds. With equation (10), for any $x, y \in B(b)$, if $k>N$, we have

$$
\begin{aligned}
\left\|T^{k} x-T^{k} y\right\|_{\varepsilon} & \leq\left(\rho\left(T^{\prime}\left(x^{*}\right)\right)+\varepsilon\right) \cdot\left\|T^{k-1} x-T^{k-1} y\right\|_{\varepsilon} \leq \cdots \\
& \leq\left(\rho\left(T^{\prime}\left(x^{*}\right)\right)+\varepsilon\right)^{k-N} \cdot\left\|T^{N} x-T^{N} y\right\|_{\varepsilon} \\
& \leq\left(\rho\left(T^{\prime}\left(x^{*}\right)\right)+\varepsilon\right)^{k-N} \cdot L_{\|\|_{\varepsilon}}\left(T, R^{n}\right)^{N} \cdot\|x-y\|_{\varepsilon} .
\end{aligned}
$$

It implies that for any positive integer $k>N$, one obtains

$$
L_{\|} \|_{\varepsilon}\left(T^{k}, B(b)\right) \leq\left(\rho\left(T^{\prime}\left(x^{*}\right)\right)+\varepsilon\right)^{k-N} \cdot L_{\|\|_{\varepsilon}}\left(T, R^{n}\right)^{N} .
$$

Since $L_{\|\|_{\varepsilon}}\left(T, R^{n}\right)<\infty$, then

$$
\begin{aligned}
\operatorname{Lip}(T, B(b)) & =\lim _{k \rightarrow \infty} L_{\|\|_{\varepsilon}}\left(T^{k}, B(b)\right)^{1 / k} \\
& \leq \lim _{k \rightarrow \infty}\left(\rho\left(T^{\prime}\left(x^{*}\right)\right)+\varepsilon\right)^{(k-N) / k} \cdot \lim _{k \rightarrow \infty} L_{\|\|_{\varepsilon}}\left(T, R^{n}\right)^{N / k} \\
& \leq\left(\rho\left(T^{\prime}\left(x^{*}\right)\right)+\varepsilon\right) .
\end{aligned}
$$

$\operatorname{Lip}(T, B(b)) \leq \rho\left(T^{\prime}\left(x^{*}\right)\right)$ holds for $\varepsilon$ is an arbitrary positive number. Thus, there exists a positive integer $N_{1}$ such that $L_{\|\|}\left(T^{k}, B(b)\right) \leq\left(\rho\left(T^{\prime}\left(x^{*}\right)\right)+\varepsilon\right)^{k}$ for the fixed $\varepsilon>0$ as a positive integer $k \geq N_{1}$. For any positive integer $k \geq N_{1}, T^{k}$ is continuously differentiable, and for any $x \in F(b) \subset B(b), x$ is an inner point of $B(b)$. By the definition of the Gateaux differential, for any $x \in F(b), k \geq N_{1}$ and $h \in R^{n}$ with $\|h\|=1$, one can obtain

$$
\begin{aligned}
\left\|\left(T^{k}\right)^{\prime}(x) \cdot h\right\| & =\lim _{t \rightarrow 0^{+}} \frac{\left\|T^{k}(x+t \cdot h)-T^{k} x\right\|}{t}=\lim _{t \rightarrow 0^{+}} \frac{\left\|T^{k}(x+t \cdot h)-T^{k} x\right\|}{\|t \cdot h\|} \\
& \leq L_{\|\|}\left(T^{k}, B(b)\right) .
\end{aligned}
$$

It implies that for any $x \in F(b)$ and $k \geq N_{1}$,

$$
\begin{aligned}
\left\|\left(T^{k}\right)^{\prime}(x)\right\| & =\sup _{\|h\|=1}\left\|\left(T^{k}\right)^{\prime}(x) \cdot h\right\| \leq L_{\|\|}\left(T^{k}, B(b)\right) \\
& \leq\left(\rho\left(T^{\prime}\left(x^{*}\right)\right)+\varepsilon\right)^{k} \leq(\alpha+\varepsilon)^{k}
\end{aligned}
$$


holds. For any $z \in R^{n}$, either $z \in F(b)$ or $z \in E(b)$, the result that if $z \in F(b)$, then $\left\|\left(T^{k}\right)^{\prime}(z)\right\| \leq \beta$ holds for any positive integer $k \geq N_{1}$ is shown above. Next, we will prove that a similar result exists for any $z \in E(b)$. For any given $z \in E(b)$ and any given positive integer $k \geq N_{1}$, there are the following two different cases.

Case 1: $T^{i} z \in E(b)$ for any positive integer $i<k$. From equation (9), one may have $\left\|\left(T^{k}\right)^{\prime}(z)\right\| \leq(\alpha+\varepsilon)^{k}$.

Case 2: There exists a positive integer $j<k$ such that $T^{i} z \in E(b)$ for any positive integer $i<j$ and $T^{j} z \in F(b)$. If $k-j \geq N_{1}$, then by the chain-rule of derivative and equations (9) and (11), we have

$$
\begin{aligned}
\left\|\left(T^{k}\right)^{\prime}(z)\right\| & =\left\|\left(T^{k-j}\right)^{\prime}\left(T^{j} z\right) \cdot\left(T^{j}\right)^{\prime}(z)\right\| \\
& \leq\left\|\left(T^{k-j}\right)^{\prime}\left(T^{j} z\right)\right\| \cdot\left\|\left(T^{j}\right)^{\prime}(z)\right\| \\
& \leq(\alpha+\varepsilon)^{k-j} \cdot(\alpha+\varepsilon)^{j} \leq(\alpha+\varepsilon)^{k} .
\end{aligned}
$$

If $k-j<N_{1}$, then from equation (9) and Lemma 1 , one gets

$$
\begin{aligned}
\left\|\left(T^{k}\right)^{\prime}(z)\right\| & =\left\|\left(T^{k-j}\right)^{\prime}\left(T^{j} z\right) \cdot\left(T^{j}\right)^{\prime}(z)\right\| \leq\left\|\left(T^{k-j}\right)^{\prime}\left(T^{j} z\right)\right\| \cdot\left\|\left(T^{j}\right)^{\prime}(z)\right\| \\
& \leq L_{\|\|}\left(T, R^{n}\right)^{k-j} \cdot(\alpha+\varepsilon)^{j} \leq(\alpha+\varepsilon)^{k} \cdot\left(\frac{L_{\|\|}\left(T, R^{n}\right)}{\alpha+\varepsilon}\right)^{k-j} .
\end{aligned}
$$

Since $\varepsilon>0$ is an arbitrary positive number, with equation (12), we have $\left\|\left(T^{k}\right)^{\prime}(z)\right\| \leq \alpha^{k}$. $\left(\frac{L_{\|\|}\left(T, R^{n}\right)}{\alpha}\right)^{k-j}$. And since $\left\|T^{\prime}(x)\right\| \leq L_{\|\|}\left(T, R^{n}\right)$ holds for any $x \in R^{n}$ (Wang et al. [9]), we have $\lambda \leq_{\|\|} L\left(T, R^{n}\right)$ and $\rho\left(T^{\prime}\left(x^{*}\right)\right) \leq L_{\|\|}\left(T, R^{n}\right)$. It implies that $\frac{L_{\|\|}\left(T, R^{n}\right)}{\alpha} \geq 1$ and $\left\|\left(T^{k}\right)^{\prime}(z)\right\| \leq$ $\alpha^{k} \cdot\left(\frac{L_{\| \Perp}\left(T, R^{n}\right)}{\alpha}\right)^{N_{1}}$.

From the above two cases, for any $k \geq N_{1}$ and $z \in E(b)$,

$$
\left\|\left(T^{k}\right)^{\prime}(z)\right\| \leq(\alpha+\varepsilon)^{k} \cdot\left(\frac{L_{\|\|}\left(T, R^{n}\right)}{\alpha}\right)^{N_{1}}
$$

By combining equations (11) and (13), one can conclude that for any positive integer $k \geq N_{1}$ and any $z \in R^{n}$,

$$
\left\|\left(T^{k}\right)^{\prime}(z)\right\| \leq(\alpha+\varepsilon)^{k} \cdot\left(\frac{L_{\|\|}\left(T, R^{n}\right)}{\alpha}\right)^{N_{1}} .
$$

For any $x, y \in R^{n}$ and $k \geq N_{1}$,

$$
L_{\|\|}\left(T^{k}, R^{n}\right)=\sup _{x, y \in R^{n}, x \neq y} \frac{\left\|T^{k} x-T^{k} y\right\|}{\|x-y\|} \leq \sup _{z \in R^{n}}\left\|\left(T^{k}\right)^{\prime}(z)\right\| \leq(\alpha+\varepsilon)^{k} \cdot\left(\frac{L_{\|\|}\left(T, R^{n}\right)}{\alpha}\right)^{N_{1}} .
$$

It implies that

$$
\operatorname{Lip}\left(T, R^{n}\right)=\lim _{k \rightarrow \infty} L_{\|\|}\left(T^{k}, R^{n}\right)^{1 / k} \leq(\alpha+\varepsilon) \cdot \lim _{k \rightarrow \infty}\left(\frac{L\left(T, R^{n}\right)}{\alpha}\right)^{\frac{N_{1}}{k}}=\alpha+\varepsilon<1 .
$$

Since $\varepsilon$ is an arbitrary positive number, $\operatorname{Lip}\left(T, R^{n}\right) \leq \alpha$ holds. 
Proof of Theorem 2 Since system (2) is of global exponential stability in $R^{n}$, there are constants $M>0$ and $0<\alpha<1$ such that for any $x_{0} \in R^{n}$ and any positive integer $k$, we have $\left\|x_{k}-x^{*}\right\| \leq M \cdot \alpha^{k} \cdot\left\|x_{0}-x^{*}\right\|$. With Lemma 1 , for any positive integer $k$ and $x \in R^{n}$, then $\left\|\left(T^{k}\right)^{\prime}\left(x^{*}\right)\right\| \leq L_{\|\|}\left(T^{k}, R^{n}\right)$. By the chain-rule of derivative, for any given positive integer $k$, one obtains $\left(T^{k}\right)^{\prime}\left(x^{*}\right)=\prod_{i=1}^{k} T^{\prime}\left(T^{k-i} x^{*}\right)=T^{\prime}\left(x^{*}\right)^{k}$. This implies that

$$
\begin{aligned}
\rho\left(T^{\prime}\left(x^{*}\right)\right) & =\lim _{k \rightarrow \infty}\left\|T^{\prime}\left(x^{*}\right)^{k}\right\|^{1 / k}=\lim _{k \rightarrow \infty}\left\|\left(T^{k}\right)^{\prime}\left(x^{*}\right)\right\|^{1 / k} \\
& \leq \lim _{k \rightarrow \infty} L_{\|\|}\left(T^{k}, R^{n}\right)^{1 / k}=\operatorname{Lip}\left(T, R^{n}\right) .
\end{aligned}
$$

$\operatorname{Lip}\left(T, R^{n}\right) \leq \alpha=\rho\left(T^{\prime}\left(x^{*}\right)\right)$ holds from Theorem 1 and $\operatorname{Lip}\left(T, R^{n}\right)=\rho\left(T^{\prime}\left(x^{*}\right)\right)$ if system (2) is globally exponentially stable in $R^{n}$. And from equation (8), for any $\varepsilon>0$ and $k$, we have

$$
\left\|T^{k} x-x^{*}\right\| \leq \frac{C(\varepsilon)_{2}}{C(\varepsilon)_{1}} \cdot\left(\operatorname{Lip}\left(T, R^{n}\right)+\varepsilon\right)^{k} \cdot\left\|x-x^{*}\right\|=\frac{C(\varepsilon)_{2}}{C(\varepsilon)_{1}} \cdot\left(\rho\left(T^{\prime}\left(x^{*}\right)\right)+\varepsilon\right)^{k} \cdot\left\|x-x^{*}\right\|,
$$

where $C(\varepsilon)_{2} \geq C(\varepsilon)_{1}>0$ are the same constants as in equation (7). It implies that $\rho\left(T^{\prime}\left(x^{*}\right)\right)$ is not less than the infimum of exponential bounds of convergent trajectories. Moreover, from Wang et al. [9], Wang and Xu [11] if system (2) is of global exponential stability in $R^{n}$ with an exponential bound $\alpha$, then $\alpha \geq \rho\left(T^{\prime}\left(x^{*}\right)\right)$. Therefore, $\rho\left(T^{\prime}\left(x^{*}\right)\right)$ is not larger than the infimum of exponential bounds of convergent trajectories. Finally, $\rho\left(T^{\prime}\left(x^{*}\right)\right)$ is the infimum of exponential bounds of convergent trajectories.

\section{Examples}

In this section, we present two examples to clarify the concepts introduced in this paper and apply the obtained results to several concrete nonlinear discrete systems.

Example 1 Consider the following recurrent neural network model studied in Jin [17]:

$$
x_{k+1}=-A x_{k}+W F\left(B x_{k}\right)+u, \quad x_{0} \in R^{n},
$$

where $x^{*} \in R^{n}$ is an equilibrium point, $A=\operatorname{diag}\left\{a_{11}, a_{22}, \ldots, a_{n n}\right\}$ is a diagonal state feedback coefficient matrix, $B=\operatorname{diag}\left\{b_{11}, b_{22}, \ldots, b_{n n}\right\}$ with $b_{i i}>0$ is the activation gain matrix; the interconnection matrix $W$ is the lower triangular given by

$$
W=\left[\begin{array}{ccccc}
w_{11} & 0 & 0 & \cdots & 0 \\
w_{21} & w_{22} & 0 & \cdots & 0 \\
w_{31} & w_{32} & w_{33} & \cdots & 0 \\
\vdots & \vdots & \vdots & \cdots & \vdots \\
w_{n 1} & w_{n 2} & w_{n 3} & \cdots & w_{n n}
\end{array}\right]
$$

$F(x)=\left(f_{1}\left(x_{1}\right), f_{2}\left(x_{2}\right), \ldots, f_{n}\left(x_{n}\right)\right)^{T}$ is the activation mapping that is diagonally nonlinear, continuously differentiable in $R^{n}$, and each $f_{i}$ satisfies $0<f_{i}^{\prime}\left(x_{i}\right)<1$ for any $x_{i} \in R^{1}$.

In Jin et al. [17], it has been shown that (14) will be of global asymptotical stability in $R^{n}$ if $\left|a_{i i}\right|+\left|w_{i i}\right| b_{i i}<1$ for any $1 \leq i \leq n$. In Wang and Xu [11], (14) is proven to be of the global exponential stability of $x^{*}$ in a bounded convergence region. Since the global exponential 
stability in the whole space is more interesting than in a bounded region, we will focus on it in the following.

Denote $T(x)=-A x+W F(B(x))+u, x \in R^{n}$. Then it is easy to see that the Jacobian matrix of $T$ at $x^{\prime \prime}$ is given by $T^{\prime}\left(x^{*}\right)=-A+W F^{\prime}\left(B\left(x^{*}\right)\right) B$ and its eigenvalues are given by

$$
-a_{i i}+w_{i i} \cdot f_{i}^{\prime}\left(b_{i i} \cdot x_{i}^{*}\right) \cdot b_{i i}: 1 \leq i \leq n .
$$

Thus, whenever $\left|a_{i i}\right|+\left|w_{i i}\right| b_{i i}<1$, we have $\rho\left(T^{\prime}\left(x^{*}\right)\right)<1$ and $\lim _{\|x\| \rightarrow \infty} \sup \left\|T^{\prime}(x)\right\|<1$; thus system (14) is of global exponential stability.

Example 2 Consider a general class of discrete-time dynamic neural networks with continuous states described in (Jin [17]) as follows:

$$
x(k+1)=-A x(k)+W \sigma[\psi x(k)]+s,
$$

where $x=\left(x_{1}, x_{2}, \ldots, x_{n}\right)^{T}$ is the neural state vector, $W=\left(w_{i j}\right)_{n \times n}$ is the synaptic weight matrix, $s=\left(s_{1}, s_{2}, \ldots, s_{n}\right)^{T}$ is the constant threshold vector, $A=\operatorname{diag}\left\{a_{1}, a_{2}, \ldots, a_{n}\right\}$ with $\left|a_{i}\right|<1$ is the feedback coefficient matrix, $\psi=\operatorname{diag}\left\{\mu_{1}, \mu_{2}, \ldots, \mu_{n}\right\}$ is the matrix of activation gains for controlling state decaying, and $\sigma(\psi x)=\left(\sigma\left(\mu_{1} x_{1}\right), \sigma\left(\mu_{2} x_{2}\right), \ldots, \sigma\left(\mu_{n} x_{n}\right)\right)^{T}$ is the vectorvalued activation function with a gain matrix $\psi$.

The nonlinear neural activation function $\sigma(\cdot)$ may be chosen as a continuous and differentiable nonlinear sigmoid function satisfying the following conditions: (1) $\sigma(x) \rightarrow \pm 1$ as $x \rightarrow \pm \infty$; (2) $\sigma(x)$ is bounded with the upper bound 1 and the lower bound -1 ; (3) $\sigma(x)=0$ at a unique point $x=0$; (4) $\sigma^{\prime}(x)>0$ and $\sigma^{\prime}(x) \rightarrow 0$ as $x \rightarrow \pm \infty$; and (5) $\sigma^{\prime}(x)$ has a global maximal value one.

Jin [17] only shows the global asymptotic stability of system (15). The global exponential stability is discussed as follows.

Denote $T(x)=-A x+W \sigma(\psi x)+s, x \in R^{n}$, then $T^{\prime}(x)=-A+W \sigma^{\prime}(\psi x) \psi$. From $\left\|T^{\prime}(x)\right\| \leq\|A\|+\left\|W \sigma^{\prime}(\psi x) \psi\right\|$ and $\sigma^{\prime}(x) \rightarrow 0$ as $x \rightarrow \pm \infty$, it is easy to know that $\lim _{\|x\| \rightarrow \infty} \sup \left\|T^{\prime}(x)\right\|<1$ as $0<\left|a_{i}\right|<1$. If $\rho\left(T^{\prime}\left(x^{*}\right)\right)<1$ is obtained at $x^{*}$, then system (15) is of global exponential stability.

Competing interests

The authors declare that they have no competing interests.

Authors' contributions

LW gave the key idea about the connection between global exponential stability and global asymptotic stability out and participating the revision and illustration. QZ participated the proof of Lemma 1 and part of Theorem 1. YF carried out the left work including the proof of Theorem 1 and Theorem 2 gave the two examples. All authors read and approved the final manuscript.

\section{Acknowledgements}

The authors would like to thank the reviewers for their valuable comments and helpful suggestions that greatly improved the note's quality.

Received: 5 May 2012 Accepted: 18 December 2012 Published: 11 January 2013

\section{References}

1. Bastinec, J, Diblik, J, Khusainov, DY, Ryvolova, A: Exponential stability and estimation of solutions of linear differential systems of neutral type with constant coefficients. Bound. Value Probl. 2010, Article ID 956121 (2010). doi:10.1155/2010/956121

2. Gordon, SP: On converse to the stability theorems for difference equations. SIAM J. Control 10, 76-81 (1972) 
3. Ortega, JM: Stability of difference equations and convergence of iterative processes. SIAM J. Numer. Anal. 10, 268-282 (1973)

4. Mousa, MS, Miller, RK, Michel, AN: Stability analysis of hybrid composite dynamical systems: descriptions involving operators and difference equations. IEEE Trans. Autom. Control 31, 603-615 (1986)

5. Jiang, ZP, Wang, Y: A converse Lyapunov theorem for discrete-time systems with disturbances. Syst. Control Lett. 45 49-58 (2002)

6. Wang, LS, Xu, ZB, Zhao, QF: Quantitative characterizations of exponential convergent property of nonlinear discrete dynamical systems. IEEE Trans. Autom. Control 52(11), 2129-2134 (2007)

7. Diblik, J, Khusainov, DY, Grytsay, IV, Smarda, Z: Stability of nonlinear autonomous quadratic discrete systems in the critical case. Discrete Dyn. Nat. Soc. 2010, Article ID 539087 (2010). doi:10.1155/2010/539087

8. Berezansky, L, Diblik, J, Ruzickova, M, Suta, Z: Asymptotic convergence of the solutions of a discrete equation with two delays in the critical case. Abstr. Appl. Anal. 2011, Article ID 709427 (2011). doi:10.1155/2011/709427

9. Wang, LS, Heng, PA, Leung, KS, Xu, ZB: Global exponential asymptotic stability in nonlinear discrete dynamical systems. J. Math. Anal. Appl. 258, 349-358 (2001)

10. Wang, LS, Xu, ZB: Sufficient and necessary conditions for global exponential stability of a class of general discrete-time recurrent neural networks. IEEE Trans. Circuits Syst. I, Regul. Pap. 53(6), 1373-1380 (2006)

11. Wang, LS, Xu, ZB: On characterizations of exponential stability of nonlinear discrete dynamical systems on bounded regions. IEEE Trans. Autom. Control 52(10), 1871-1881 (2007)

12. Meyers, PR: A converse to Banach's contraction theorem. J. Res. Natl. Bur. Stand. 71(B), 73-76 (1967)

13. Leader, S: A topological characterization of Banach contractions. Pac. J. Math. 69(2), 461-466 (1977)

14. Opoitsev, VI: A converse to the principle of contracting maps. Russ. Math. Surv. 31(4), 175-204 (1976)

15. Soderlind, G: On nonlinear difference and differential equations. BIT Numer. Math. 24, 667-680 (1984)

16. Elaydi, SN: An Introduction to Difference Equations. Springer, New York (1995)

17. Jin, L, Gupta, MM: Global asymptotic stability of discrete-time analog neural networks. IEEE Trans. Neural Netw. 7(6), 1024-1031 (1996)

\section{Submit your manuscript to a SpringerOpen ${ }^{\ominus}$ journal and benefit from:}

- Convenient online submission

- Rigorous peer review

- Immediate publication on acceptance

- Open access: articles freely available online

- High visibility within the field

- Retaining the copyright to your article 